\title{
Editorials
}

\section{Is the practice of haematology evidence based? III. Evidence based diagnostic testing}

In our two previous articles we have discussed whether there is an evidence based approach to the clinical practice of haematology and the morphological interpretation of blood films and bone marrows. ${ }^{12}$ In this final article we will discuss the need for evidence based diagnostic testing in haematology.

One of the main challenges facing modern health care is the increasing demand placed on providers of services. There is evidence that diagnostic testing is a key driver in increasing health care costs. In one study in the USA, the relation between the number of diagnostic tests performed and the therapeutic interventions which they might trigger was examined. ${ }^{3}$ Data were collected from Medicare's National Claims History. The results of this study showed that an increasing use of diagnostic tests resulted in an increase in related therapeutic intervention rates. Clearly, for some patients detection of disease would result in an appropriate therapeutic treatment. For other patients, however, early detection of disease may not be clinically important and the question then of whether the therapeutic intervention is appropriate or not arises. Therefore, as pointed out by Muir Gray, ${ }^{4}$ if attempts are made to control the volume of treatment without attempting to control the use of diagnostic services then this strategy will undoubtedly result in failure. Within this debate, commissioners of health care will be aiming to ensure that diagnostic tests are used only in situations where clinically appropriate therapeutic interventions are offered. Haematologists also have an important role to play in ensuring that laboratory resources are used effectively in the diagnostic process.

As defined in the Strategic review of pathology, ${ }^{5}$ haematologists, like all pathologists, should be involved both in the process of producing information contained in a pathology report and also in influencing demand for pathology services. As far as developing an evidence based approach to diagnosis is concerned, five steps have been identified. ${ }^{6}$ These include:

- Gathering clinical findings

- Framing clinical problems

- Selecting a differential diagnosis

- Choosing a diagnostic test

- Interpreting test results.

Haematologists wearing their laboratory hat have an important role to play in the last two steps in this process (and in the context of liaison haematology, which we are not covering in these articles, in the first three steps as well).

When choosing a laboratory test, users require information on the evaluation of diagnostic tests. There is clear published guidance for methods that should be used when evaluating a diagnostic test. ${ }^{7}$ The range of methods includes validation of the test against a gold standard, selection of populations used to define a reference range, definition of precision and accuracy of the test, reproducibility, and so on. Users also need to know whether a test has been placed in the context of other potential tests in the diagnostic sequence for the condition under investigation. The American College of Physicians has published diagnostic strategies for various areas including the investigation of anaemia, erythrocytosis, and coagulation disorders. ${ }^{8}$ Finally, users will require guidance in the final step of the diagnostic process in how they interpret the results of laboratory tests. While individual haematologists will be comfortable with interpreting results of haematology tests, many users need further guidance. This is particularly true since few laboratory results are either simply positive or negative; there is usually a gradual change in results from what is thought to be normal to what may be abnormal. Therefore the way in which a normal range has been defined is clearly an important factor when assessing the usefulness of any diagnostic test. A common definition of normal assumes that the diagnostic test results of a normal population fit a normal or Gaussian distribution. By defining a normal range as the mean plus or minus two standard deviations, this will include $95 \%$ of its population. This leaves $2.5 \%$ at each of its upper and lower ends who, by definition, are then abnormal and are labelled with a diagnosis of disease. However, as discussed by Sackett and colleagues, ${ }^{9}$ there are several problems with this approach. For example, if the highest and lowest $2.5 \%$ of diagnostic test results are called abnormal, then all diseases will have the same frequency. Another problem with this approach is that not all diagnostic test results will fit a Gaussian distribution. (This issue of how the term "normal" is defined is further discussed by Sackett and colleagues. ${ }^{9}$ ) As a result it has been suggested that either likelihood ratios or number needed to diagnose (NND) may be a useful alternative way of expressing results of laboratory tests.

Likelihood ratios express the odds that a given level of a diagnostic test result would be expected in a patient with the target disorder. The likelihood ratios can be derived from the sensitivity and specificity of tests at various cut off points. For example, in table 1 we have reproduced data which were adapted from an overview of 55 studies of the usefulness of serum ferritin in the diagnosis of iron deficiency anaemia. As can be seen from the table there is no clear cut off value between normal and abnormal.

For each serum ferritin concentration, likelihood ratios have been calculated. Using a published nomogram (fig 1) the likelihood ratio can be used together with the prevalence (pre-test probability) of a disorder to determine the post-test probability of that disorder being present. ${ }^{10}$ For example, studies have shown that $36 \%$ of patients aged over 65 years who are anaemic have iron deficiency. ${ }^{11}$ Using the nomogram the post-test probability of iron deficiency can then be determined depending on the ferritin result. As can be seen from the nomogram, a ferritin concentration of less than $5 \mu \mathrm{mol} /$ litre increases the probability of iron deficiency being present from $36 \%$ (pre-test probability) to over $95 \%$ (post-test probability). Conversely, a ferritin level of more than $95 \mu \mathrm{mol} /$ litre reduces the probability of iron 
Table 1 Likelihood ratios and number needed to diagnose for five serum ferritin concentrations

\begin{tabular}{|c|c|c|c|c|c|c|}
\hline \multirow{2}{*}{$\begin{array}{l}\text { Serum ferritin } \\
\text { ( } \text { momol/litre) }\end{array}$} & \multicolumn{2}{|c|}{ Patients with iron deficiency } & \multicolumn{2}{|c|}{ Patients free of iron deficiency } & \multirow[b]{2}{*}{ Likelihood ratiof } & \multirow{2}{*}{$\begin{array}{l}\text { Number needed to } \\
\text { diagnose } \neq\end{array}$} \\
\hline & Number & Proportion (sensitivity) & Number & Proportion (1 - specificity) & & \\
\hline$<15$ & 474 & 0.5859 & 20 & 0.0113 & 52 & 1.7 \\
\hline $15-34$ & 175 & 0.2163 & 79 & 0.0446 & 4.8 & 5.8 \\
\hline $35-64$ & 82 & 0.1014 & 171 & 0.0966 & 1.0 & 208 \\
\hline $65-94$ & 30 & 0.0371 & 168 & 0.0949 & 0.39 & -17 \\
\hline$\geqslant 95$ & 48 & 0.0593 & 1332 & 0.7525 & 0.08 & -1.4 \\
\hline
\end{tabular}

Data in columns 1 to 6 have been reproduced with permission from Sackett DL, Haynes RB, Guyatt GH, Tugwell P: Clinical epidemiology. A basic science for clinical medicine. Boston: Little, Brown and Co, 1991:130.

†Likelihood ratio $=$ sensitivity $/(1-$ specificity $)$.

$\ddagger$ Number needed to diagnose $=1 /[$ sensitivity $-(1-$ specificity $)]$

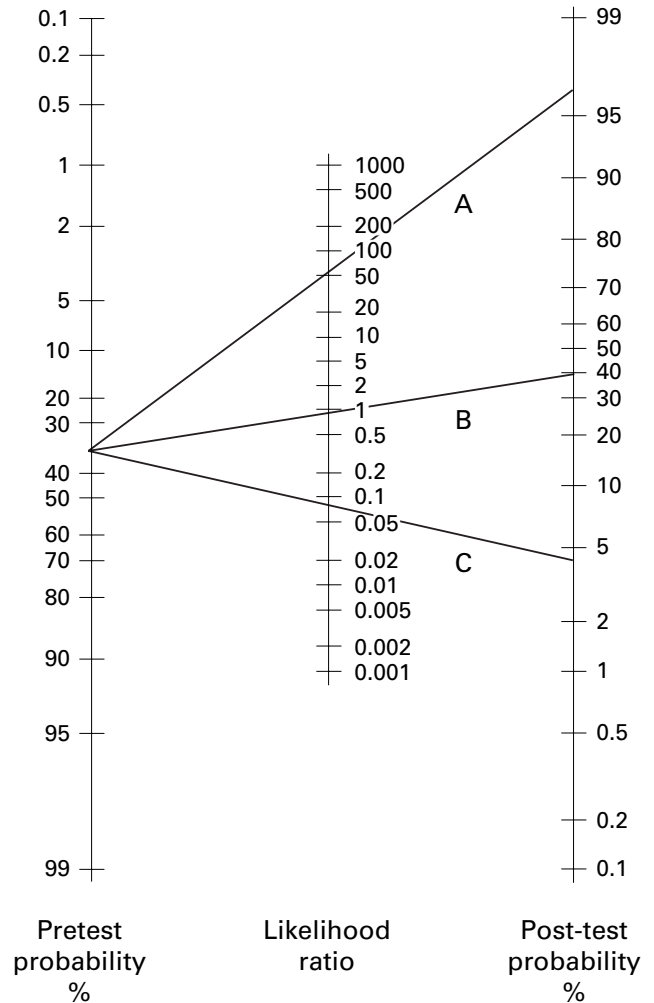

Figure 1 Use of likelihood ratios to calculate post-test probability of iron deficiency at three different serum ferritin concentrations. $A,<5 ; B, 35-64$; $C,>95$ umol/litre.

deficiency being present to below 5\%. An intermediate level of ferritin - that is, between 35 and $64 \mu \mathrm{mol} /$ litre-has little impact on the probability of iron deficiency being present.

Although initially the use of likelihood ratios appears to be more complicated than the more familiar reference ranges, it does have the advantage of being able to produce useful information regarding the value of diagnostic tests at different cut off points for test results. However, even in this rather specific area of haematology different nomograms would be needed for children, pregnant women, men aged less than 65 years, and so on. The scale of the information required to generate pre-test probability could be a significant barrier to developing this sort of approach, as would be the large number of nomograms required by those interpreting the test results.

Another approach to the evaluation of diagnostic tests is the use of the NND, which is again also derived from the sensitivity and specificity of a test. ${ }^{12}$ One of the proposed advantages of this NND is that it also allows for comparison of costs of laboratory tests. ${ }^{13}$ The NND is the number of tests which is required in order to gain a positive diagnosis. As a comparison the NND for the presence of iron deficiency using ferritin results has been calculated (table 1). As can be seen, with increasing ferritin concentrations the NND increases. However, once ferritin is above $65 \mu \mathrm{mol} /$ litre a minus value is derived for this calculation. It would therefore appear that where the likelihood ratio is less than 1, a negative value for NND is obtained. NND may have a role for haematologists in comparing the cost-effectiveness of laboratory tests, particularly if there is more than one test which can be used in a specific diagnostic situation. This application of NND may be limited by the problem of obtaining a negative value in situations where a diagnostic test performs poorly.

Therefore likelihood ratios, and to a lesser extent NND, may be helpful to users in the interpretation of diagnostic tests. The application of this approach would appear to be limited to specific diagnostic circumstances within laboratory haematology. In the example given above, the situation was one of assessing the value of ferritin in the diagnosis of iron deficiency. Many laboratory tests, particularly full blood counts, are performed in situations where clinicians may be either screening for disease when the result is expected to be normal or monitoring progress rather than trying to obtain a specific diagnosis. It is difficult to see how this approach would be helpful in these clinical circumstances. Users will usually need to rely either on reference ranges or changes from previous results in the same patient when interpreting results in such circumstances.

In conclusion then, it would appear that the use of likelihood ratios may improve the ability of users to interpret laboratory results in some specific situations. Further work is needed in this area to determine whether likelihood ratios can then result in a more effective use of laboratory services. Expansion of this approach to cover a wide range of haematology test results does raise the practical issue of implementation. One strategy is for the haematologist to take the lead in developing the information which can be included in the format of a laboratory report. As mentioned above, however, the application of likelihood ratios may only be appropriate in a small area of laboratory haematology. It is probably too much to expect that clinical users of laboratories will maintain up to date nomograms for each test for which they require information. Therefore this approach will remain a challenge to all haematologists if they are to develop a format in which to present laboratory data which are accessible to users and which help them determine post-test probabilities when considering use of laboratory tests. It is our experience that this aspect of evidence based practice is at best embryonic and in most cases pre-conceptional!

M J GALLOWAY

Department of Haematology,

Bishop Auckland Hospitals NHS Trust,

Bishop Auckland, Co Durham, UK

M M REID

Department of Haematology,

Royal Victoria Infirmary and

Associated Hospitals NHS Trust,

Newcastle upon Tyne, Northumberland, UK 
1 Galloway MJ, Reid MM. Is the practice of haematology evidence based? I. Evidence based clinical practice. $\mathcal{F}$ Clin Pathol (in press).

2 Reid MM, Galloway MJ. Is the practice of haematology evidence based? II. Evidence based morphological diagnosis. F Clin Pathol (in press).

3 Verrilli D, Welch HG. The impact of diagnostic testing on therapeutic investigations. $\mathcal{F A M A}$ 1996;275:1189-91.

4 Gray JAM. Evidence-based healthcare. Edinburgh: Churchill Livingstone, 1997:34-45.

5 NHS Executive. Strategic review of pathology services. London: HMSO, 1995.

6 Richardson WS. Evidence-based diagnosis: more is needed. Evidence-Based Medicine 1997;2:70-1.

7 Greenhalgh T. How to read a paper. The basics of evidence based medicine. London: BMJ Publishing Group, 1997:102-10.
8 Panzer RJ, Black ER, Griner PF. Diagnostic strategies for common medical problems. Philadelphia: American College of Physicians, 1991.

9 Sackett DL, Haynes RB, Guyatt GH, et al. Clinical epidemiology. A basic science for clinical medicine. Boston: Little, Brown and Co, 1991:51-68.

10 Sackett DL, Haynes RB, Guyatt GH, et al. Clinical epidemiology. A basic science for clinical medicine. Boston: Little, Brown and Co, 1991:119-30.

11 Guyatt GH, Patterson C, Ali M, et al. Diagnosis of iron deficiency anaemia in the elderly. Am $\mathcal{F}$ Med 1990;88:205-9.

12 How good is that test, II? Bandolier 1996 May;3(5):1-3.

13 Batstone G. Practising by the evidence: the role of pathology. $\mathcal{F}$ Clin Pathol 1997;50:447-9.

\section{The polymerase chain reaction - a time of transition from research to routine}

The identification of hepatitis B virus (HBV) as the cause of "serum hepatitis" followed the discovery of a new protein (Australia antigen) in blood samples and its later recognition as a viral surface protein (HBsAg). Detection of HBsAg remains the front line diagnostic assay for hepatitis $\mathrm{B}$, both in the routine virology laboratory and for screening donated blood, and most infected individuals also are positive for antibodies to the nucleocapsid protein (anti-HBc). However, not all $\mathrm{HBsAg}$ carriers have a significant viraemia: HBsAg may be produced from $\mathrm{HBV}$ DNA and integrated into the genomes of the hepatocytes without virus replication. The situation is confounded further by the fact that some individuals who are believed to have recovered from infection, with seroconversion to antiHBs, may have very low levels of virus - detectable by the polymerase chain reaction $(\mathrm{PCR})^{1}-$ which may reactivate in the face of immunosuppression.

Detection of hepatitis B viraemia is facilitated by another protein, $\mathrm{HBeAg}$, which is secreted by the infected hepatocytes. $\mathrm{HBeAg}$ in serum invariably indicates ongoing virus replication in the liver. The converse, however, does not necessarily apply, and HBeAg may be absent from - and its antibody (anti-HBe) present in-viraemic individuals, especially those infected with precore, core promoter, and some other HBV mutants. Hepatitis B viraemia can only be established unequivocally by the detection of the genome. HBV DNA was first detected indirectly by assaying the endogenous polymerase activity and, following its cloning and sequencing, directly by a variety of hybridisation assays and the PCR.

In the February issue, van Duersen et al reported their evaluation of the PCR to resolve diagnostic uncertainties following serological testing of five individuals for markers of HBV infection. ${ }^{2}$ The detection of HBsAg in a blood donor five days after hepatitis $B$ immunisation is reassuring, as this testifies to the exquisite sensitivity of current assays. The fear for this front line assay is that HBsAg with amino acid sequence variation in the major antigenic determinant may escape detection, especially by assays which rely on monoclonal antibodies for antigen capture or detection. Such variants have been described most often in immunised children ("vaccine failures") and in liver transplant recipients treated with hepatitis B immune globulin in an attempt to prevent infection of the graft, but may be detected as minority species in persistently infected individuals and selected during natural seroconversion to anti-HBs.

Of the four remaining patients tested, ${ }^{2}$ one was HBsAg negative but gave a false positive result when tested for HBeAg. This patient was from a high risk group for HBV infection and a negative PCR could itself cause a diagnos- tic dilemma: if sequences encoding HBsAg are variant, the primer binding sites may be too. As van Duersen and his colleagues point out, PCR protocols and their performance in detecting HBV DNA are not yet standardised for general use. Three patients were positive using the PCR; one was also HBsAg positive and of interest for the absence of anti$\mathrm{HBc}$, which was not pursued. A patient with a single change in the immunodominant region of HBsAg illustrates the paradox that polyclonal antibody may be more likely than monoclonal antibody based assays to fail to detect variant HBsAg with single amino acid substitutions, ${ }^{3}$ especially where the antigen is present in low titre. The final case, with multiple substitutions in HBsAg, illustrates the real threat to surface antigen testing. The concern is that failure to detect HBsAg will lead, not to diagnostic dilemmas, but to the use of a donated unit of blood which proves to be HBV positive.

Despite a long standing reluctance to introduce assays which detect nucleic acid into routine diagnostic laboratories, it seems inevitable that assays based on the PCR will be used more and more in such laboratories. The recently discovered GB virus $\mathrm{C}^{4}$ (GBV-C, also known as hepatitis G virus) is a case in point. While antibodies to the viral surface protein (anti-E2) seem to be a marker of recovery, attempts to devise assays for antigens or antibodies present during infection have not been successful, and diagnosis of viraemia depends on detecting the genomic RNA. But for the lack of evidence of pathogenicity, it seems inevitable that routine PCR testing of donated blood for GBV-C would already have been introduced. However, experience with another recently discovered virus, HHV-8 or KSHV, illustrates all too well that even established research laboratories may fall foul of PCR contamination. ${ }^{5}$

To comply with an EC requirement from 1 January 1999, PCR testing of plasma pools used for blood products must begin for hepatitis $C$ virus in the near future. The aim is to detect those rare donations in the window period between infection and seroconversion to anti-HCV. Testing of "minipools" of around 500 donations seems the most likely scenario, and there will be an obligation to analyse positive pools to identify the source. One suspects that pressure will follow to test for HIV and other viruses (for example, HBV and parvovirus, perhaps in a multiplex format) and, later, to test blood used for individual transfusions.

I thank Peng Lee Yap of the Scottish Blood Transfusion Service for helpful discussions.

TIM J HARRISON†

Chester Beatty Laboratories,

Institute of Cancer Research,

London SW3 6FB, UK 
tOn sabbatical leave from the Royal Free Hospital School of Medicine.

1 Rehermann B, Ferrari C, Pasquinelli C, et al. The hepatitis B virus persists for decades after patients' recovery from acute viral hepatitis despite active maintenance of a cytotoxic T-lymphocyte response. Nature Med maintenance 8 ; 2 : $1104-8$.

2 van Duersen FJ, Hino K, Wyatt D, et al. Use of PCR in resolving diagnostic dilemmas due to genetic variation of hepatitis B. 7 Clin Pathol 1998;51:149-54.

3 Bruce SA, Murray K. Mutations of some critical amino acid residues in the hepatitis B virus surface antigen. $\mathcal{F}$ Med Virol 1995;46:157-61.

4 Simons JN, Leary TP, Dawson GJ, et al. Isolation of novel virus-like sequences associated with human hepatitis. Nature Med 1995;1:564-9.

5 Moore PS. Human herpesvirus 8 variants. Lancet 1998;351:679-80.

\section{CALL FOR COVER ILLUSTRATIONS}

Readers with material that might be suitable for the front cover of the Journal are invited to submit prints or transparencies to the editor. Description and acknowledgement is published inside the front cover. This could be a good way of getting your work seen! 